\title{
Jointly Optimized Bit-Rate/Delay Control Policy for Wireless Packet Networks With Fading Channels
}

\author{
Javad Razavilar, Member, IEEE, K. J. Ray Liu, Senior Member, IEEE, and Steven I. Marcus, Fellow, IEEE
}

\begin{abstract}
In this paper, we consider the downlink rate control problem in a wireless channel. A dynamic programming optimization method is introduced to obtain the optimal bit-rate/delay control policy in the downlink for packet transmission in wireless networks with fading channels. We assume that the base station is capable of transmitting data packets in the downlink with different bit rates, $R_{0}<R_{1}<\cdots<R_{M-1}$. It is assumed that the symbol rate is fixed in the system, and different bit rates are achieved by choosing the transmitted symbols from the appropriate signal constellation (adaptive modulation). The derived optimal rate control policy, in each time slot, selects the highest possible bit rate which minimizes the delay and at the same time minimizes the number of rate switchings in the network. The optimal bit-rate control problem is an important issue, especially in packet data networks, where we need to guarantee a quality of service $(\mathrm{QoS})$ in the network. Our analytical as well as simulation results confirm that there is an optimal threshold policy to switch between different rates.
\end{abstract}

Index Terms-Adaptive modulation, dynamic programming, optimal bit-rate control, wireless packet networks.

\section{INTRODUCTION}

$\mathbf{T}$ HE INCREASING popularity of the wireless network services with limited amount of available resources calls for highly efficient resource allocation methods [1], [2]. One of the major issues in wireless data networks is the bit-rate control problem [2], [3]. This is especially important in the downlink, since in a wireless data network most of the traffic flow is from the base station to mobiles, e.g., an Internet connection or a multimedia (voice/image/data) connection. A good rate control algorithm has a great impact on the network performance.

In this paper, we investigate the rate control problem for wireless channels from an optimal control point of view. There exists some literature on obtaining the nature of optimal control policies for a wide range of related problems [1], [4]-[8]. In [5], Lambadaris and Narayan have considered the problem of jointly optimal admission and routing at a data network node. Another good example is [9], which deals with optimal control of service in tandem queues. In [10], the authors consider the problem of stochastic control of handoffs in cellular networks and try to find an optimal policy for the handoff problem.

Paper approved by K. K. Leung, the Editor for Wireless Network Access and Performance of the IEEE Communications Society. Manuscript received August 19, 1999; revised January 17, 2001.

J. Razavilar is with Magis Networks, Inc., San Diego, CA 92130 USA (e-mail: jrazavilar@magisnetworks.com).

K. J. R. Liu and S. I. Marcus are with the Electrical and Computer Engineering Department and Institute for Systems Research, University of Maryland, College Park, MD 20742 USA (e-mail: marcus@eng.umd.edu).

Publisher Item Identifier S 0090-6778(02)02035-4.
In this paper, we derive some properties of a class of optimal rate control-problems using the theory of dynamic programming (DP). The general nature of the problem considered is as follows. The base station transmits the data packets over a wireless channel to mobile users (downlink problem). We assume that the base station is capable of transmitting data packets with different bit rates, $R_{0}<R_{1}<\cdots<R_{M-1}$. It is assumed that the symbol rate is fixed in the system and different bit rates are achieved by choosing the transmitted symbols from the appropriate signal constellations (adaptive modulation). The received signal-to-noise ratio (SNR) by the mobile users is subject to fluctuation due to fading and noise. In our system model, we assume that no power control mechanism is in effect in the downlink, and the base station transmits the packets always with its maximum available power. Therefore, each user can be served with highest possible bit rate, depending on channel condition, and only the rate-control algorithm determines the appropriate bit rate for each user. This would relieve the system from complexities of the power control algorithm also. This is an important advantage of our proposed system model which has clear benefits in practical systems.

We assume a finite-state Markov model (FSMM) for the wireless channel. The mobile constantly monitors the received SNR. At each measurement instant, the mobile observes the state of the channel and determines the current channel state. At each decision making instant by employing an optimal strategy, the mobile decides whether to send a request to the base station to switch the rate for the next time slot or not. To facilitate this, the system needs a feedback channel (assumed to be noise-free) so the mobile terminal can send its requests to the base station. If there are $M$ admissible rates in the system, then we require a $\log _{2} M$-bit feedback channel. For example, in a system with two admissible rates $R_{0}$ and $R_{1}$, the feedback channel needs to be only one bit. Fig. 1 illustrates the block diagram of a system where the mobile employs an optimal strategy in choosing the rate in the network.

The optimal policy which determines the choice of rates (or modulation schemes) should try to use the highest possible rate which minimizes the delay in sending the packets and at the same time minimizes the number of rate switchings. We show that under certain conditions the optimal strategy has the form of a threshold policy. Intuitively, it makes sense that, for very low SNRs, packets are transmitted in the downlink with the lowest bit rate $R_{0}$ (e.g., transmitted symbols are chosen from a QPSK constellation) and at very high SNRs packets are transmitted in the downlink with the highest bit rate $R_{M-1}$ (e.g., transmitted symbols are chosen from a 64-QAM constellation).

A properly designed rate control algorithm would result in high data transmission quality (low delay) and low signaling 


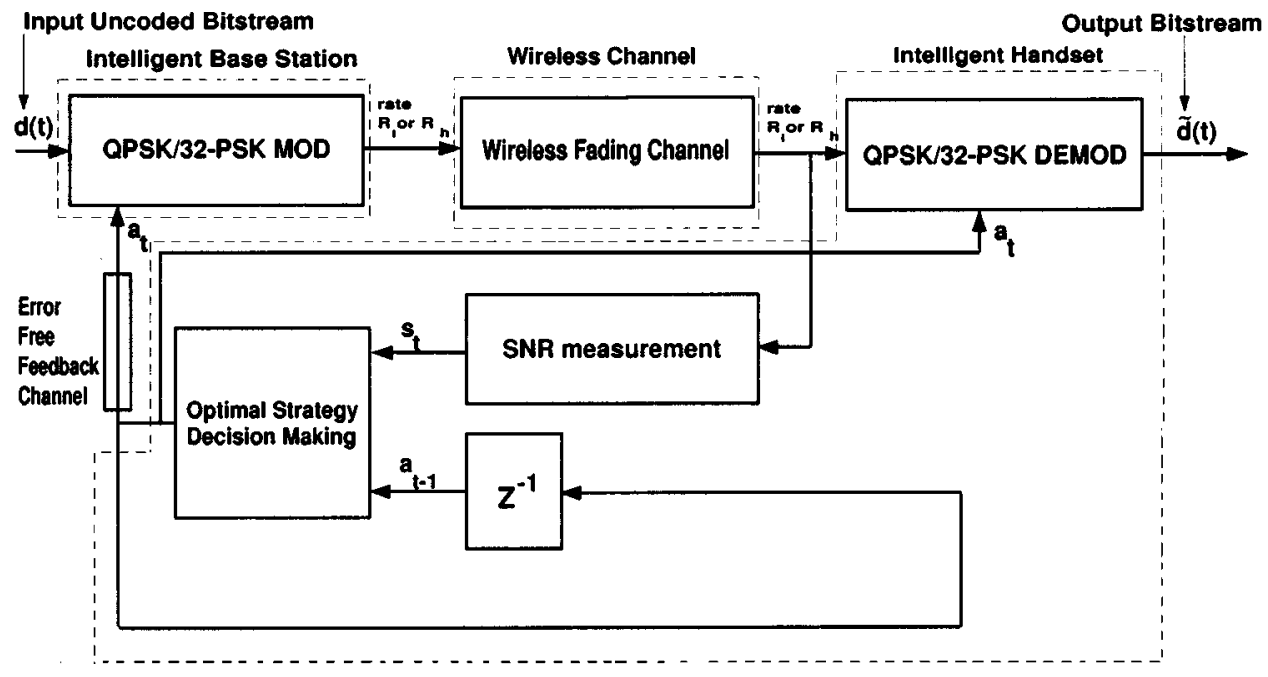

Fig. 1. Block diagram of a system where the mobile employs an optimal strategy in choosing the data rate in the network.

and switching costs. In a wireless fading channel, signal strength fluctuates due to multipath. Because of the statistical SNR fluctuations, the bit rate during a data transmission session needs to be switched between different admissible rates ranging from $R_{0}$ to $R_{M-1}$. In the absence of an optimal rate control policy, this may cause unnecessary and frequent rate switchings which results in protocol overheads (due to rate negotiation process). If decisions are made solely on the basis of the "lowest delay" without any penalty for switching the rate, then the "chattering" effect may develop. It is clear that if there is no switching cost for switching from one rate to the other, then the optimal rate switching policy is trivial, which is to switch to the rate with the smallest delay. On the other hand, delaying rate switching decisions, as the signal strength received from the base station starts to deteriorate, may result in the termination of the data transmission in the middle of a session (due to the SNR dropping below the minimum acceptable threshold defined by the standard). A sluggish policy which delays rate switching decisions for too long will result in a high probability of forced termination.

Therefore, the objective of the rate control algorithm must be to minimize the number of rate switchings while maintaining the minimum delay in transmitting the packets. This calls for an efficient rate switching algorithm which can capture a tradeoff between data transmission quality and switching cost, in an appropriately balanced manner.

The paper is organized as follows. In Section II, a finite-state Markov channel model for wireless Rayleigh fading channels is presented. Section III reviews some of the relevant results from the theory of dynamic programming. The optimal data rate control problem cast as an infinite horizon discounted cost dynamic programming problem forms the subject of Section IV. The average delay of transmitting the packets and the expected number of rate switchings and also a practical method to choose a reasonable value for the rate switching cost are studied in Section V. Simulation results are presented in Section VI. Finally, Section VII includes our conclusions and remarks.

\section{MARKov MODEL FOR WiRELESS CHANNELS}

The study of the finite-state Markov channel (FSMC) emerges from early work of Gilbert [11] and Elliot [12]. They study a two-state Markov channel known as the Gilbert-Elliot channel. In their channel model, each state corresponds to a specific channel quality which is either noiseless or totally noisy. In cases when the channel quality varies dramatically, modeling a radio channel as a two-state Gilbert-Elliot channel is not adequate. This is the case for urban wireless fading channels. The idea is to form a finite-state Markov model for such wireless channels [13]-[15]. Let $\mathcal{S}=\{0,1, \ldots, K-1\}$ denote a finite set of states. By partitioning the range of the received SNR into a finite number of intervals, FSMC models can be constructed for Rayleigh fading channels [13]-[15]. The members of set $\mathcal{S}$ correspond to those partitions. Now let $\left\{S_{n}\right\}, n=0,1, \ldots$ be a stationary Markov process. Since a stationary Markov process has the property of time-invariant transition probabilities, the transition probability is independent of the time index $n$ and can be written as

$$
\begin{gathered}
p_{s s^{\prime}} \equiv \operatorname{Pr}\left(S_{n+1}=s^{\prime} \mid S_{n}=s\right), \quad n=0,1, \ldots, s \\
s^{\prime} \in\{0,1, \ldots, K-1\} .
\end{gathered}
$$

If we assume that the transitions only happen between adjacent states, we obtain

$$
p_{s s^{\prime}}=0, \quad\left|s-s^{\prime}\right|>1, s, s^{\prime} \in\{0,1, \ldots, K-1\}
$$

In a typical multipath propagation environment, the received signal envelope has the Rayleigh distribution. With additive Gaussian noise, the received instantaneous SNR $\gamma$ is distributed exponentially with probability density function (PDF)

$$
p(\gamma)=\frac{1}{\gamma_{0}} \exp \left(-\frac{\gamma}{\gamma_{0}}\right), \quad \gamma \geq 0
$$

where $\gamma_{0}$ is the average SNR. An FSMC model can be built to represent the time-varying behavior of the Rayleigh fading channel. We start by partitioning the received SNR into a finite number of intervals. Let $\Gamma_{0}=0<\Gamma_{1}<\Gamma_{2} \cdots<\Gamma_{K}=\infty$ be the thresholds of the received SNR. Then the channel is in state $k$ if the received SNR is between $\Gamma_{k}$ and $\Gamma_{k+1}$. For a 

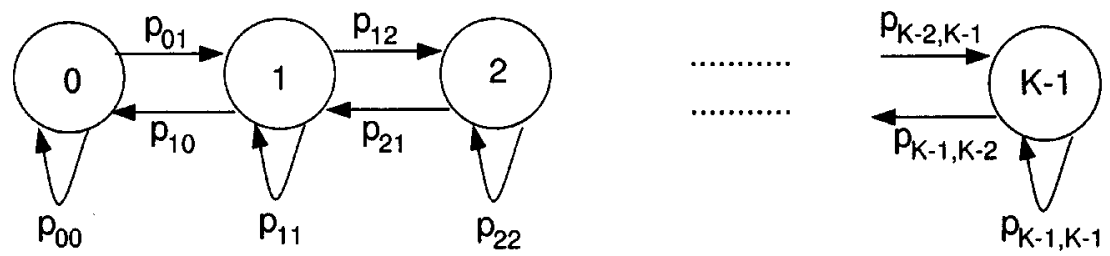

Fig. 2. $\quad K$-state noisy channel with Markov transitions modeling a Rayleigh fading channel

packet transmission system, we assume that a one-step transition in the model corresponds to the channel state transition after one packet time period $T_{p}$. A received packet is said to be in channel state $s_{k}, k=0,1, \ldots, K-1$, if the SNR values in the packet varies in the range $\left[\Gamma_{k}, \Gamma_{k+1}\right)$. In this case, the steady-state probabilities of the channel states are given by

$$
\pi_{k}=\int_{\Gamma_{k}}^{\Gamma_{k+1}} p(\gamma) d \gamma, \quad k=0,1, \ldots, K-1
$$

In this FSMC model, we allow transitions from a given state to its two adjacent states only. The transition probabilities $p_{s s^{\prime}}$ in Fig. 2 can be determined using the following equations [14], [15]:

$$
\begin{aligned}
& p_{k, k+1}=\frac{N\left(\Gamma_{k+1}\right) T_{p}}{\pi_{k}}, \quad k=0,1, \ldots, K-2 \\
& p_{k, k-1}=\frac{N\left(\Gamma_{k}\right) T_{p}}{\pi_{k}}, \quad k=1,2, \ldots, K-1
\end{aligned}
$$

where $T_{p}=L_{p} / R_{s}$ is the packet transmission time, where $R_{s}$ is the symbol rate in the system and $L_{p}$ is the packet size (in our system model both $R_{s}$ and $L_{p}$ are fixed for all modulation schemes). In (5) and (6), $N($.$) is the level crossing function$ given by

$$
N(\Gamma)=\sqrt{\frac{2 \pi \Gamma}{\gamma_{0}}} f_{d} \exp \left(-\frac{\Gamma}{\gamma_{0}}\right)
$$

where $f_{d}$ is the maximum Doppler frequency defined as

$$
f_{d}=\frac{\nu}{\lambda}
$$

where $\nu$ is the mobile's speed, and $\lambda$ is the wavelength. Equations (5) and (6) are used in Section VI to compute the transition probabilities of the FSMC model. From this model, we proceed to obtain an optimal policy for the rate control problem over Rayleigh fading wireless channels.

\section{DYNAMIC PROGRAMMING}

In this section, we review some of the relevant results from the theory of dynamic programming [16]-[19] which will be used subsequently to derive the nature of optimal policies for a class of rate control problems. The stochastic model of the wireless channel is such that the states of the underlying Markov model of the channel evolve according to a time-invariant Markov transition rule independent of past and present rate control decisions made by the mobile. Let $\left\{S_{t}, t=0,1, \ldots\right\}$ be a discrete time process. At any given time, the state of the channel $S_{t}$ takes its value from a finite-state space denoted by the set of nonnegative integers $\{0,1, \ldots, K-1\}$. In our problem, this set represents the finite-state space of the underlying Markov model of the channel. At the beginning of the time slot $[t, t+1$ ), for $t=0,1, \ldots$, the channel is in state $s$ and the packets are transmitted in the downlink with rate $R_{i}$ and a decision must be made as to which rate to select for transmitting the packets in the downlink during the time slot $[t, t+1)$. Let $U_{t}$ denote the $\{0,1, \ldots, M-1\}$-valued random variable which encodes the decision taken at time $t$, i.e., if $U_{t}=i, i=0,1, \ldots, M-1$, then the rate $R_{i}$ will be used during the time slot $[t, t+1)$. We set $I_{t} \equiv U_{t-1}$ which denotes the bit rate at which the packets are transmitted during the time slot $[t-1, t)$. Now let us define the aggregate state of the system as $\left(S_{t}, I_{t}\right)$ which takes values in $\{0,1, \ldots, K-1\} \times\{0,1, \ldots, M-1\}$. Suppose that for time slot $\left[t, t+1\right.$ ) the mobile chooses the action (rate) $U_{t}$ while the aggregate state of the system is $\left(S_{t}, I_{t}\right)$. Then we incur an instantaneous cost $R\left(S_{t}, I_{t}, U_{t}\right)$, which is a bounded mapping from the finite space $R:\{0,1,2 \ldots, K-1\} \times\{0,1, \ldots, M-$ $1\} \times\{0,1, \ldots, M-1\} \mapsto \mathbb{R}$, where $\mathbb{R}$ denotes the set of real numbers. We define a Markov policy, $\pi$, as a mapping for choosing the sequence of decisions $U_{t}, t=0,1, \ldots$ Therefore, a policy $\pi$ is a mapping from the aggregate state space to the action space, i.e., $\pi:\{0,1,2 \ldots, K-1\} \times\{0,1, \ldots, M-1\} \mapsto$ $\{0,1, \ldots, M-1\}$. Given the evolution of the aggregate state of the system $\left\{S_{t}, I_{t}\right\}_{t=0}^{\infty}$, we are interested in the solution of the following problem. Choose $\pi$ such that

$$
V^{\pi}(s, i) \equiv E_{(s, i)}^{\pi}\left[\sum_{t=0}^{\infty} \beta^{t} R\left(S_{t}, I_{t}, U_{t}\right)\right]
$$

is minimized, where $(s, i)$ is the initial state of the system, and $E_{(s, i)}^{\pi}$ denotes the expectation under the policy $\pi$, with $I_{0}$ being arbitrary, and $0<\beta \leq 1$ is the discount factor. This problem is called an infinite horizon discounted cost problem. The above cost reflects the fact that, while choosing the rate $u_{t}$ for time slot $[t, t+1)$, we would like to take into account the effect of this decision on the future behavior of the system. For the case where $0<\beta<1$, the use of the discount factor is motivated by the fact that a cost to be incurred in the future is less important than one incurred at the present time instant.

It is important to mention that $\beta$ has a nice practical meaning in the system. A session initiated at time $t=0$ will last a random number $T$ of time slots. We may interpret $1-\beta$ as the probability that a session is terminated in a time slot and therefore $\beta$ is the probability that a session continues in a time slot. Consequently, session duration random variable $T$ is geometrically distributed with

$$
P[T=t+1]=(1-\beta) \beta^{t}
$$




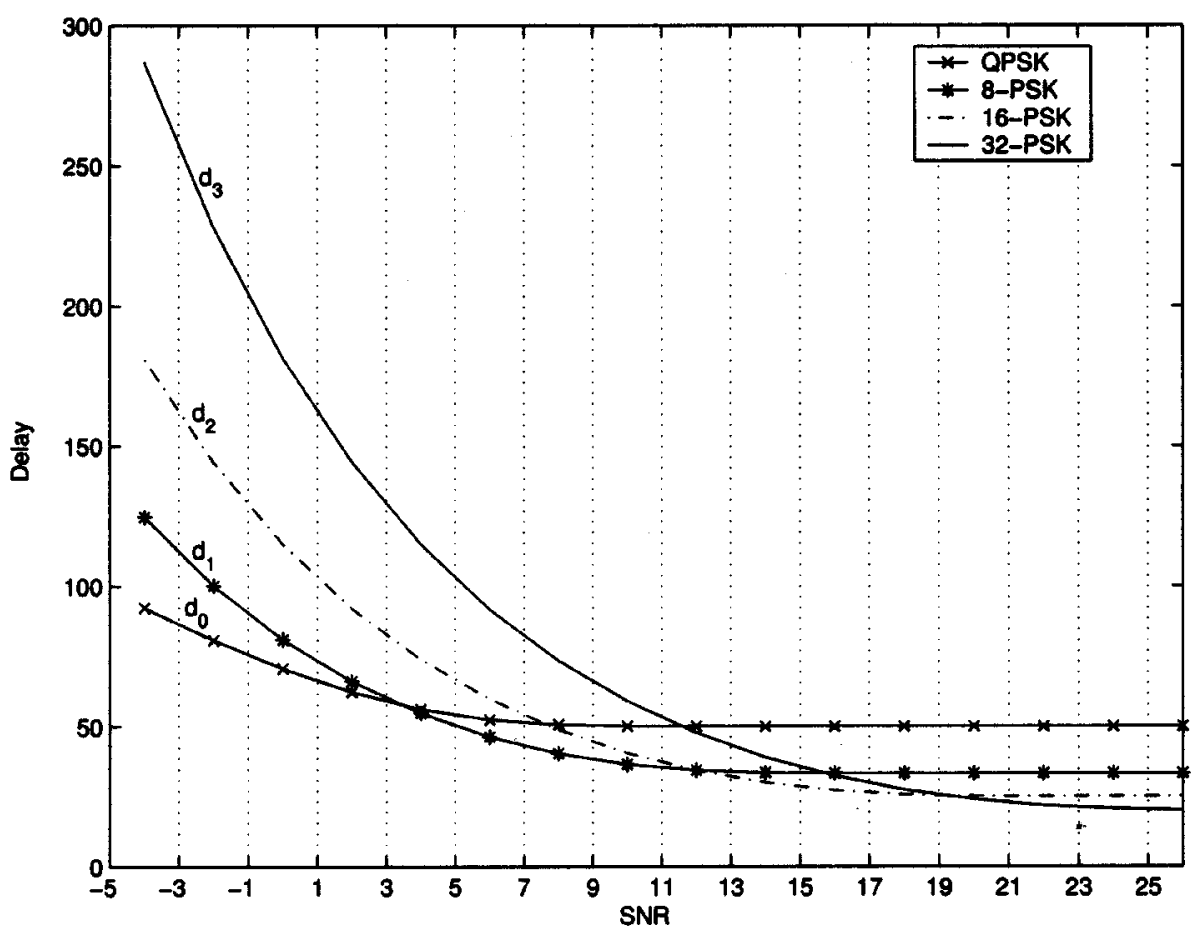

Fig. 3. Delay functions $d_{0}, d_{1}, d_{2}$, and $d_{3}$ in a multirate system with four admissible rates $R_{0}, R_{1}, R_{2}$, and $R_{3}$ (for four different modulation schemes QPSK, 8-PSK, 16-PSK, and 32-PSK, respectively) versus SNR.

\section{Optimal Data Rate Control}

In order to formulate the optimal rate control problem as a stochastic optimization problem, we need to define a cost structure which quantifies the cost associated with operating the system under any policy. Obviously there is no unique way of defining the cost function. The choice of cost function clearly affects the optimization problem and the structure of the optimal policy. We attempt to choose the cost function in such a way that the incurred cost makes physical sense in the actual network.

\section{A. Cost Function Structure for Optimal Rate Control}

In this section, we introduce a cost function which captures the desired tradeoff between data transmission quality and switching cost, in an appropriate balanced manner for the optimal rate control (allocation) problem. First we need to introduce a transmission quality metric. We define the transmission delay functions $d_{m}:\{0,1, \ldots, K-1\} \rightarrow R$, $m=0,1, \ldots, M-1$, as follows [20]:

$$
d_{m}(s) \equiv \frac{L_{p}}{R_{m} T_{s}\left(1-P_{e m}(s)\right)}, \quad m=0,1, \ldots, M-1
$$

where $L_{p}$ is the packet size in symbols per packet, $R_{m}$ is the bit rate in bits per second (b/s), $T_{s}$ is the symbol time in seconds (which is fixed in the system), $R_{m} T_{s}$ is in $\mathrm{b} / \mathrm{s} / \mathrm{Hz}$ which represents the number of bits transmitted per symbol (a.k.a. spectral efficiency, for example for 32-ary PSK modulation scheme, $R_{m} T_{s}=5$ ), and $P_{e m}$ is the symbol error rate (SER)

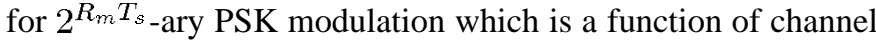
state (symbol SNR) [21]. Fig. 3 illustrates delay functions $d_{0}$, $d_{1}, d_{2}$, and $d_{3}$ for four different modulation schemes QPSK, 8-PSK, 16-PSK, and 32-PSK, in a multirate system with four admissible rates $R_{0}, R_{1}, R_{2}$, and $R_{3}$, respectively. As expected, at very low SNRs, QPSK has the minimum delay among these four modulation schemes, and at very high SNRs 32-PSK has the minimum delay response.

In order to have a reasonable cost-per-stage $R$ each time the mobile unit switches from one rate to another, this should be penalized by a cost associated with rate switching. Let $C_{s}$ denote the cost of the rate switching. On the other hand, a reward (which is a function representing the transmission quality) encourages the mobile unit to switch the rate in order to minimize the delay in the network.

Let $d_{i}(s)$ denote the delay for the current rate and $d_{j}(s)$ denote the delay for the candidate rate to switch to. If $d_{i}(s) \leq$ $d_{j}(s)$, then $d_{i}(s)-d_{j}(s)$ represents the reward for not switching the rate but if $d_{i}(s)>d_{j}(s)$, then $d_{i}(s)-d_{j}(s)$ represents the missed opportunity for improving the transmission quality (reducing the transmission delay) by not switching the rate. Since there is a cost $C_{s}$ associated with switching the rate, our optimal rate control policy captures a tradeoff between data transmission quality and switching cost, in an appropriate balanced manner. In a multirate system, we must ensure that if the optimal policy chooses to switch the rate at a given state of the system, then it must select the rate which has the minimum delay among all admissible rates. From Fig. 3, it is clear that, for any given SNR and current rate $i$ with delay $d_{i}(s)$, the best candidate rate $j$ with delay $d_{j}(s)$ to switch to is the one that $\max _{\{j \neq i, j=0,1, \ldots, M-1\}}\left(d_{i}(s)-d_{j}(s)\right)$. The "max" operator here forces the optimal policy to switch to the rate with minimum delay among all admissible rates, if it decides to switch the rate (given the state of the system). For example, in Fig. 3, at low SNRs the optimal policy must choose to stay with rate $R_{0}$ with associated delay $d_{0}(s)$. In this case, as SNR increases, 
the best candidate rate to switch to would be rate $R_{1}$ with associated delay $d_{1}(s)$, and the reward (or missed opportunity to improve the call quality) for staying with rate $R_{0}$ would be equal to $d_{0}(s)-d_{1}(s)$. This is consistent with our proposed reward function that, for the given rate, it selects the maximum delay difference with other available rates in the system as the reward for staying with the current rate. Having discussed the appropriate structure of the cost function for our optimal rate control problem, we are now ready to introduce the cost-per-stage function $R($.$) as follows:$

$$
R(s, i, u)= \begin{cases}C_{s}, \max _{\{j=1,2, \ldots, M-1\}} & \text { if } i \neq u \\ \left.\operatorname{mat}_{i}(s)-d_{i \ominus j}(s)\right], & \text { if } i=u\end{cases}
$$

with $\oplus$ denoting modulo $M$ addition and $i=0,1, \ldots, M-1$. For the special case where $M=2$, (12) for $i=u$ reduces to $d_{0}-d_{1}$ when $i=u=0$ and to $d_{1}-d_{0}$ when $i=u=1$. Therefore, if there are only two admissible rates $R_{0}$, and $R_{1}$ in the system, then the cost function $R($.$) is simplified to$

$$
R(s, i, u)= \begin{cases}C_{s}, & \text { if } i \neq u \\ (-1)^{i}\left(d_{0}(s)-d_{1}(s)\right), & \text { if } i=u .\end{cases}
$$

Now the problem at hand is to solve the following infinite horizon discounted cost problem:

$$
V(s, i) \equiv \min _{u=0,1, \ldots, M-1} E_{s, i}^{\pi}\left[\sum_{t=0}^{\infty} \beta^{t} R(s, i, u)\right]
$$

for every $(s, i)$ in $\{0,1,2, \ldots, K-1\} \times\{0,1, \ldots, M-1\}$ and policy $\pi$. In order to ensure the existence of the expected infinite horizon discounted cost, it suffices to have a uniformly bounded cost function $R\left(S_{t}, I_{t}, U_{t}\right)$ for all $t \in\{0,1, \ldots\}$ and $0<\beta<1$. In our rate control problem, the state and action spaces are finite, $\left|R\left(S_{t}, I_{t}, U_{t}\right)\right|<B<\infty$ for $\forall t, \in$ $\{0,1, \ldots\}$, and with the interpretation of $\beta$ in a practical system we always have $0<\beta<1$. This set of conditions ensures existence of a solution for our optimal rate control problem. The policy $\pi$ satisfying the problem cast in (14) is called the optimal policy $\pi^{*}$. Below we state a well-known result [17], [18] which yields an implicit equation satisfied by the optimal discounted cost function $V(s, i)$.

Theorem 1: $V($.$) satisfies the optimality equation:$

$$
\begin{aligned}
& V(s, i)=\min _{u} {\left[R(s, i, u)+\beta \sum_{s^{\prime}} p_{s s^{\prime}} V\left(s^{\prime}, u\right)\right], } \\
&(s, i) \in\{0,1, \ldots, K-1\} \times\{0,1, \ldots, M-1\}
\end{aligned}
$$

where $(s, i)$ is the initial state of the system, and $p_{s s^{\prime}}$ is the state transition probability of the finite-state Markov model of the wireless channel given by set of equations (5) and (6). In effect, (15) provides that the cost incurred by choosing an action $u$ at some time instant is the sum of the instantaneous cost $R(s, i, u)$, and the expected cost for the future $\sum_{s^{\prime}} p_{s s^{\prime}} V\left(s^{\prime}, u\right)$ multiplied by the given discount factor $\beta$. The optimal policy chooses that action $u$ which minimizes this sum.
In the sequel, we attempt to find the solution of (15) using an iterative method. For this purpose, we define the following quantity:

$$
\tilde{V}_{n-1}(s, i) \equiv \sum_{s^{\prime}} p_{s s^{\prime}} V_{n-1}\left(s^{\prime}, i\right)
$$

Then the DP equation is simply

$$
V_{n}(s, i)=\min _{\{u=0,1, \ldots, M-1\}}\left\{R(s, i, u)+\beta \tilde{V}_{n-1}(s, u)\right\} .
$$

Equations (12), (16), and (17) are used in our computer simulations to find the solution for (15) in the general case. This method is called value iteration or successive approximation. In order to understand the structure of optimal policy, from now on, we would like to restrict our attention to the mathematically more tractable case. Therefore, without loss of generality, in the following we only consider the case where $M=2$ and the set of admissible rates is $u \in\{0,1\}$ (corresponding to $R_{0}$ and $R_{1}$ ). In this case, (17) can be rewritten as

$$
\begin{aligned}
V_{n}(s, i)=\min & \left\{C_{s}+\beta \tilde{V}_{n-1}(s, i \oplus 1),\right. \\
& \left.(-1)^{i}\left(d_{0}(s)-d_{1}(s)\right)+\beta \tilde{V}_{n-1}(s, i)\right\}
\end{aligned}
$$

where $\oplus$ denotes modulo two addition. Moreover, the optimal policy $\pi^{\star}$ is a Markov stationary policy which selects to switch in state $(s, i)$ if and only if

$C_{s}+\beta \tilde{V}_{n-1}(s, i \oplus 1) \leq(-1)^{i}\left(d_{0}(s)-d_{1}(s)\right)+\beta \tilde{V}_{n-1}(s, i)$.

An important observation regarding the solution of the discounted DP problem given by (17) is that it can be interpreted as the fixed point of a well-defined operator $T$ where $T V=V$. Motivated by the form of the dynamic programming equation (17), we associate $R$-valued mappings $\tilde{T} \varphi$ and $T_{u} \varphi, u=0,1$ defined on $\{0,1,2, \ldots, K-1\} \times\{0,1\}$ by setting

$$
(\tilde{T} \varphi)(s, i) \equiv \sum_{s^{\prime}} p_{s s^{\prime}} \varphi\left(s^{\prime}, i\right)
$$

and

$$
\left(T_{u} \varphi\right)(s, i) \equiv R(s, i, u)+\beta(\tilde{T} \varphi)(s, u)
$$

for $(s, i) \in\{0,1,2, \ldots, K-1\} \times\{0,1\}$. Next, we introduce the operator $T$ by setting

$$
\begin{aligned}
(T \varphi)(s, i) \equiv \min _{u=0,1}\left(T_{u} \varphi\right)(s, i), & \\
& (s, i) \in\{0,1,2, \ldots, K-1\} \times\{0,1\}
\end{aligned}
$$

for every $\varphi$. Now, using the important properties given in [10], [17], and [18], we state the following important results.

Proposition 1: Under the model assumptions [stationary Markov model for the channel, bounded cost-per-stage function $R($.$) , and 0<\beta<1$, where $1-\beta$ is the probability of terminating a session in a time slot], the following statements hold. 
1) The operator $T$ is a strict contraction mapping.

2) The value function $V$ is the only solution of the fixed point equation

$$
\varphi=T \varphi .
$$

3) Moreover, for every element $\varphi$, the recursive scheme

$$
\varphi_{0}=\varphi, \quad \varphi_{k+1}=T \varphi_{k}, \quad k=0,1, \ldots
$$

converges to the value function $V$ in the sense that $\lim _{k}\left\|\varphi_{k}-V\right\|_{2}=0$, where $\lim _{k} \varphi_{k}(s, i)=V(s, i)$ for all $(s, i) \in\{0,1,2, \ldots, K-1\} \times\{0,1\}$.

Now we will use the results of the theorems given in [10], [17], and [18] to investigate the structure of the optimal policy. In fact, it turns out that the optimal rate control policy $\pi^{*}$ belongs to the class of threshold policies. A rate switching policy $\pi$ is said to be a threshold policy with threshold functions $\tau_{i} i=0,1$, if it is a Markov stationary policy such that

$$
\pi^{\star}(s, 0)=1 \quad \text { iff } z(s) \geq \tau_{0}
$$

and

$$
\pi^{\star}(s, 1)=0 \quad \text { iff } z(s) \leq \tau_{1}
$$

where $z(s) \equiv d_{0}(s)-d_{1}(s)$, with $z:\{0,1,2, \ldots, K-1\} \rightarrow$ $R$. First we use Fig. 3 in an attempt to illustrate the optimal threshold policy given by (25) and (26). From Fig. 3, we focus our attention to the intersection point of the delay curves $d_{0}$ and $d_{1}, s_{0}$ with $d_{0}\left(s_{0}\right)-d 1\left(s_{0}\right)=0$. The optimal threshold policy (25) simply states that if the current rate is $R_{0}$, then there exist a $\tau_{0}$, and an $s \geq s_{0}$ such that the $d_{0}(s) \geq d_{1}(s)+\tau_{0}$ and the rate must be switched from $R_{0}$ to $R_{1}$. Otherwise, if the current rate is $R_{1}$, the optimal threshold policy (26) states that there exist a $\tau_{1}$ and an $s \leq s_{0}$ such that $d_{0}(s) \leq d_{1}(s)+\tau_{1}$ and the rate must be switched from $R_{1}$ to $R_{0}$. Now we are ready to discuss the following important result about the structure of the optimal rate control policy for the problem at hand.

Proposition 2: Under the model assumptions (stationary Markov model for the channel, bounded cost-per-stage function $R($.$) , and 0<\beta<1$, where $1-\beta$ is the probability of terminating a session in a time slot), the optimal rate control policy $\pi^{\star}$ is a threshold policy with thresholds $\tau_{i}^{\star} \in R, i=0,1$, which are uniquely determined through the equations

$$
C_{s}+(-1)^{i} \beta(\Delta \tilde{T} V)\left(\tau_{i}^{\star}\right)=(-1)^{i} \tau_{i}^{\star}, \quad \tau_{i}^{\star} \in R, i=0,1 .
$$

Furthermore, $\tau_{1}^{\star} \leq \tau_{0}^{\star}$.

Proof: Fix $(s, i)$ in $\{0,1, \ldots, K-1\} \times\{0,1\}$, and $z=$ $d_{0}(s)-d_{1}(s)$ in $R$. We begin by rewriting the dynamic programming equation (18) in the following form:

$$
\begin{aligned}
V(z, i)=\min \left\{C_{s}+\beta(\tilde{T} V)(z, i \oplus 1),\right. & \\
& \left.(-1)^{i} z+\beta(\tilde{T} V)(z, i)\right\} .
\end{aligned}
$$

The optimal policy $\pi^{\star}$ is the Markov stationary policy which selects to switch in state $(s, i)$ if and only if

$$
C_{s}+\beta(\tilde{T} V)(z, i \oplus 1) \leq(-1)^{i} z+\beta(\tilde{T} V)(z, i)
$$

or, equivalently, if and only if

$$
C_{s}+(-1)^{i} \beta(\Delta \tilde{T} V)(z) \leq(-1)^{i} z, \quad z \in R
$$

where

$$
(\Delta \tilde{T} V)(z)=(\tilde{T} V)(z, 1)-(\tilde{T} V)(z, 0) .
$$

For $i=0(i=1)$, the left-hand side of the inequality (28) is a monotone nonincreasing (nondecreasing) function of $z$, while its right-hand side is a strictly increasing (decreasing) function of $z$. It is now a simple matter to conclude that the switching sets $S_{i}(z) \equiv\left\{z \in R: C_{s}+(-1)^{i} \beta(\Delta \tilde{T} V)(z) \leq(-1)^{i} z\right\}, i=$ 0,1 , are nonempty closed and connected sets which are disjoint (owing to the condition $C_{s}>0$ ). In fact, $S_{0}(z)=\left[\tau_{0}^{\star}, \infty\right)$ with $\tau_{0}^{\star}=\inf S_{0}(z)$, and $S_{1}(z)=\left(-\infty, \tau_{1}^{\star}\right]$ with $\tau_{1}^{\star}=\sup S_{1}(z)$, and the optimal policy is of threshold type. Because $S_{0}(z)$ and $S_{1}(z)$ are disjoint sets, we see that $\tau_{1}^{\star} \leq \tau_{0}^{\star}$ and this concludes the proof of Proposition 2.

It is easy to see that the method described in Proposition 2, under a certain condition, can be used in multirate systems also to determine the optimal rate switching thresholds in such systems. In fact, in multirate systems, $C_{s}$ must be upper bounded by a positive number $C_{\max }$, i.e., $0 \leq C_{s} \leq C_{\max }$ to be able to use (27) in such systems. $C_{\max }$ depends on system parameters, and it can be easily determined through simulations. We use Fig. 3 to illustrate how the optimal thresholds can be determined in a multirate system, using Proposition 2. For example, for the delay curves $d_{1}$ and $d_{2}$ associated with rates $R_{1}$ and $R_{2}$ in Fig. 3, given that $0 \leq C_{s} \leq C_{\max }$, one can apply (27) to these two delay functions to determine the optimal thresholds to switch between $R_{1}$, and $R_{2}$. This point will become clear in Section VI where we provide our simulation results for the optimal threshold policy in a multirate system.

\section{Average Delay and Rate Switchings}

Once a rate control (allocation) policy (be it optimal or not) has been selected, it is of interest to compute the average delay of transmitting the packets over the wireless channel and the expected number of rate switchings that the mobile experiences while the optimal policy is in effect. These two quantities constitute good measures of the effectiveness of a rate control policy.

We define the average delay $D_{\pi}$ of the policy $\pi$ to be the mean value of the delay of the selected rate to receive the packets from the base station under the policy $\pi$ during the packet transmission, namely

$$
D_{\pi}(s, i) \equiv \mathbf{E}_{s, i}^{\pi}\left[\sum_{t=0}^{\infty} \beta^{t}\left(I_{t} d_{1}\left(S_{t}\right)+\left(1-I_{t}\right) d_{0}\left(S_{t}\right)\right)\right] .
$$

On the other hand, the expected number of rate switchings under the policy $\pi$ is defined by

$$
S_{\pi}(s, i) \equiv \mathbf{E}_{s, i}^{\pi}\left[\sum_{t=0}^{\infty} \beta^{t} \mathbf{1}\left[I_{t} \neq U_{t}\right]\right]
$$

where $\mathbf{1}($.$) is the indicator function and it is equal to one if the$ condition (.) is met. Therefore both $D_{\pi}$ and $S_{\pi}$ can be written 
as discounted cost functions. For any Markov stationary policy $\pi$, and in particular for any threshold policy, this fact can be exploited for numerical purposes by interpreting $D_{\pi}$ and $S_{\pi}$ as fixed points for suitably defined contraction mappings. More precisely, to evaluate the average delay, for each Markov stationary policy $\pi$, we consider an operator $K_{\pi}$ of the form

$$
\left(K_{\pi} \varphi\right)(s, i) \equiv\left(K_{\pi(s, i)} \varphi\right)(s, i)
$$

for every $(s, i) \in\{0,1,2, \ldots, K-1\} \times\{0,1\}$, where for each $u=0,1$ the operator $K_{u}$ is defined by

$$
\left(K_{u} \varphi\right)(s, i) \equiv d_{i}(s)+\beta(\tilde{T} \varphi)(s, u) .
$$

As in Proposition 1, the operator $K_{u}, u=0,1$, is a contraction mapping and so is $K_{\pi}$. It follows from the Markov property that the average delay $D_{\pi}$ is the unique fixed point of $K_{\pi}$ and can be evaluated through the recursion

$$
\varphi_{0}=0, \quad \varphi_{k+1}=K_{\pi} \varphi_{k}, \quad k=0,1, \ldots
$$

To compute the expected number of rate switchings, we use the operator $K_{\pi}^{\star}$ which is of the form

$$
\left(K_{\pi}^{\star} \varphi\right)(s, i) \equiv\left(K_{\pi(s, i)}^{\star} \varphi\right)(s, i)
$$

for every $(s, i) \in\{0,1,2, \ldots, K-1\} \times\{0,1\}$, where for each $u=0,1$, the operator $K_{u}^{\star}$ is defined by

$$
\left(K_{u}^{\star} \varphi\right)(s, i) \equiv \mathbf{1}[u \neq i]+\beta(\tilde{T} \varphi)(s, u) .
$$

This time, the operators $K_{u}^{\star}, u=0,1$, are contraction mappings, and so is $K_{\pi}^{\star}$. The unique fixed point of $K_{\pi}^{\star}$ is $S_{\pi}$ and is obtained through the recursion

$$
\varphi_{0}=0, \quad \varphi_{k+1}=K_{\pi}^{\star} \varphi_{k}, \quad k=0,1, \ldots
$$

It is clear that both $D_{\pi}(s, i)$ and $S_{\pi}(s, i)$ are functions of channel state $s \in\{0,1, \ldots, K-1\}$ and rate $i \in\{0,1, \ldots, M-1\}$. It would be useful to calculate the average delay and average switching rate over all possible channel states and admissible rates for a fixed $C_{s}$. Therefore, we have

$$
\begin{aligned}
\bar{D}_{\pi} & =\sum_{i^{\prime}=0}^{M-1} p_{i^{\prime}} \sum_{s^{\prime}=0}^{K-1} \pi_{s^{\prime}} D_{\pi}\left(s^{\prime}, i^{\prime}\right) \\
\bar{S}_{\pi} & =\sum_{i^{\prime}=0}^{M-1} p_{i^{\prime}} \sum_{s^{\prime}=0}^{K-1} \pi_{s^{\prime}} S_{\pi}\left(s^{\prime}, i^{\prime}\right)
\end{aligned}
$$

where $\pi_{s}$ is the steady-state probability given by (4), and $p_{i}$ is the probability of selecting rate $R_{i}$ under the adopted rate control policy and channel model, i.e., $p_{i}=\operatorname{Pr}\left[I_{t}=i\right], i=$ $0,1, \ldots, M-1$. For the special case where $M=2$, we have $p_{0}=1-p_{1}$, and the threshold policy given by (25) and (26) can be rewritten as

$$
U_{t}=I_{t+1}= \begin{cases}1, & \text { if } Z_{t} \geq \tau_{0} \\ I_{t}, & \text { if } \tau_{1} \leq Z_{t} \leq \tau_{0} \\ 0, & \text { if } Z_{t} \leq \tau_{1} .\end{cases}
$$

Using (40) and the i.i.d. assumption on the random variables $\left\{Z_{t}, t=0,1, \ldots\right\}$, it can be shown that the sequence of
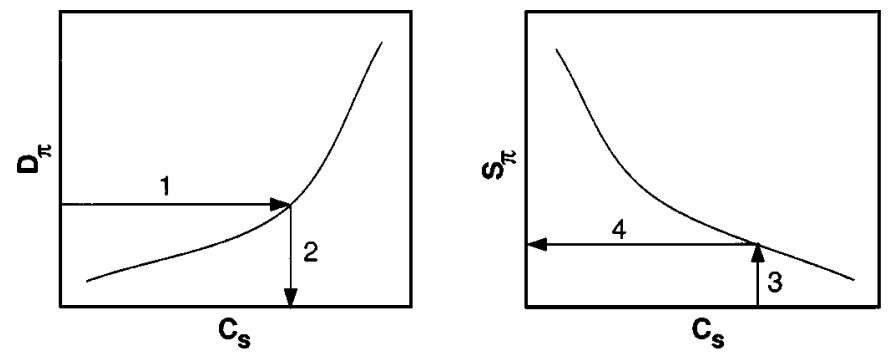

Fig. 4. A typical graph for $D_{\pi}$ and $C_{\pi}$ versus switching $\operatorname{cost} C_{s}$.

random variables $\left\{I_{t}, t=0,1, \ldots\right\}$ form a Markov chain on $\{0,1\}$ with the following transition probabilities:

$$
\begin{aligned}
& P_{01}=P\left[I_{t+1}=1 \mid I_{t}=0\right]=P\left[Z_{t} \geq \tau_{0}\right] \\
& P_{10}=P\left[I_{t+1}=0 \mid I_{t}=1\right]=P\left[Z_{t} \leq \tau_{1}\right] .
\end{aligned}
$$

Using (41) and (42), we obtain

$$
p_{0}=P\left[I_{t}=0\right]=\frac{P_{10}}{P_{01}+P_{10}} .
$$

Now (43) can be used along with (39) to compute $\bar{D}_{\pi}$ and $\bar{S}_{\pi}$ for a fixed $C_{s}$. Intuitively, as $C_{s}$ increases, the average delay $\bar{D}_{\pi}$ increases while the expected number of rate switchings $\bar{S}_{\pi}$ decreases. Our simulation results in Section VI confirms this observation.

\section{A. A Heuristic Approach for Selecting the Switching Cost}

One of the critical parameters on which the optimal rate control policy clearly depends is the value of the rate switching $\operatorname{cost} C_{s}$. Given a rate switching $\operatorname{cost} C_{s}$, we can compute an optimal rate control policy which solves the minimization problem posed in (14). Based on our previous discussion, $\bar{D}_{\pi}$ is an increasing function of $C_{s}$, while $\bar{S}_{\pi}$ is a decreasing function of $C_{s}$, similar to the graphs shown in Fig. 4. As a design procedure, we can start from a desired value of $\bar{D}_{\pi}$ and use the graph in Fig. 4 (the actual graph is obtained in Section VI) to find the respective value of $C_{s}$ and from there the respective value of $\bar{S}_{\pi}$. If the resulting value of $\bar{S}_{\pi}$ is satisfactory, then the rate control policy is acceptable, otherwise the procedure has to be restarted by choosing a larger $\bar{D}_{\pi}$. The above mentioned procedure is summarized by a flowchart in Fig. 5 .

\section{Simulation RESUltS}

In the preceding sections, we studied the rate control problem in wireless networks and offered a novel method based on DP to obtain an optimal rate control policy. In this section, our simulations results for the solution of rate control problem posed in (15) are presented. In these simulations, successive approximation method (a.k.a., value-iteration method) is used to solve (15). Our simulation results indicate that the optimal strategy for selecting the rates is indeed a threshold policy. This corroborates the results of Proposition 2 presented in the previous section.

The wireless channel is modeled as a finite-state Markov chain [14], [15]. The parameters for the simulations are as follows: symbol rate $R_{s}=500 \mathrm{Ks} / \mathrm{s}$, packet size $L_{p}=200$ symbols (i.e., $T_{p}=0.4 \mathrm{~ms}$ ), and Doppler frequency $f_{d}=10$ Hz. We consider a 15-state Markov model for the fading channel, with average SNR $\gamma_{0}=12 \mathrm{~dB}$, and SNR thresholds 


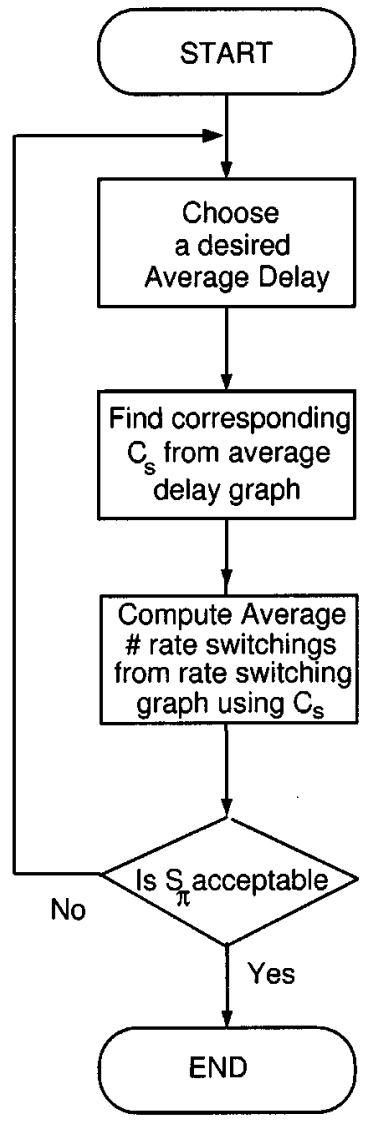

Fig. 5. Flowchart of the design procedure to select an appropriate switching $\operatorname{cost} C_{s}$.

$\Gamma_{1}=-2 \mathrm{~dB}$ and $\Gamma_{i}=\Gamma_{i-1}+\Delta, i=2,3, \ldots, 14$, where $\Delta=2 \mathrm{~dB}$. Table I shows the state transition and steady-state probabilities for this channel. Table I entries are calculated using (4)-(6).

First we consider the optimal rate control policy in a system with two admissible rates, i.e., $M=2$. In this case we assume that packets are transmitted in the downlink using either a QPSK modulation or a 16-PSK modulation (i.e., $R_{0}=2 R_{\mathrm{s}} \mathrm{b} / \mathrm{s}$, and $R_{1}=4 R_{s} \mathrm{~b} / \mathrm{s}$, where $R_{s}$ is the symbol rate). The simulation results for optimal thresholds for two values of $C_{s}$ are shown in Fig. 6. These optimal thresholds along with the transmission delay curves, $d_{0}$ and $d_{1}$, are plotted in the same figure for comparison purposes. The optimal policy $\pi^{\star}(s, i)$ for $C_{s}=45$, plotted in Fig. 6, is illustrated in Table II. The optimal policy $\pi^{\star}(s, i)$, essentially dictates the rate to be used if the system is in state $(s, i)$. This notion is demonstrated in a matrix form given by Table II.

As we discussed earlier, if there is no cost for switching the rates, i.e., $C_{s}=0 \mathrm{in}$ (13), the optimal policy for the rate control problem is simply to switch to the rate with smaller delay. Therefore, in this case, the optimal thresholds $\tau_{0}$ and $\tau_{1}$ are equal to zero $\left(\tau_{0}=\tau_{1}=0\right)$. Our simulations results confirms this observation. Fig. 6 illustrates this case, which is obtained for $C_{s}=0$ in the cost function given by (13). The optimal threshold rate control policy is also obtained for $C_{s}=45$ which is demonstrated in Fig. 6. It is worth mentioning that, as $C_{s}$ increases, $\tau_{0}-\tau_{1}$ increases as well, in other words, the optimal policy
TABLE I

State Transition and Steady-State Probabilities of the FSMC With $T_{p}=0.4 \mathrm{~ms}, f_{d}=10 \mathrm{~Hz}, \mathrm{AND} \gamma_{0}=12 \mathrm{~dB}$

\begin{tabular}{c||c|c|c|c}
\hline state index $k$ & $\pi_{k}$ & $p_{k, k-1}$ & $p_{k, k}$ & $p_{k, k+1}$ \\
\hline \hline 0 & $3.9029 \mathrm{e}-002$ & - & $9.5074 \mathrm{e}-001$ & $4.9258 \mathrm{e}-002$ \\
\hline 1 & $2.2118 \mathrm{e}-002$ & $8.6920 \mathrm{e}-002$ & $8.0617 \mathrm{e}-001$ & $1.0691 \mathrm{e}-001$ \\
\hline 2 & $3.4016 \mathrm{e}-002$ & $6.9512 \mathrm{e}-002$ & $8.4615 \mathrm{e}-001$ & $8.4340 \mathrm{e}-002$ \\
\hline 3 & $5.1405 \mathrm{e}-002$ & $5.5810 \mathrm{e}-002$ & $8.7792 \mathrm{e}-001$ & $6.6269 \mathrm{e}-002$ \\
\hline 4 & $7.5556 \mathrm{e}-002$ & $4.5087 \mathrm{e}-002$ & $9.0318 \mathrm{e}-001$ & $5.1735 \mathrm{e}-002$ \\
\hline 5 & $1.0629 \mathrm{e}-001$ & $3.6778 \mathrm{e}-002$ & $9.2325 \mathrm{e}-001$ & $3.9974 \mathrm{e}-002$ \\
\hline 6 & $1.3951 \mathrm{e}-001$ & $3.0455 \mathrm{e}-002$ & $9.3917 \mathrm{e}-001$ & $3.0376 \mathrm{e}-002$ \\
\hline 7 & $1.6420 \mathrm{e}-001$ & $2.5808 \mathrm{e}-002$ & $9.5173 \mathrm{e}-001$ & $2.2463 \mathrm{e}-002$ \\
\hline 8 & $1.6291 \mathrm{e}-001$ & $2.2642 \mathrm{e}-002$ & $9.6148 \mathrm{e}-001$ & $1.5882 \mathrm{e}-002$ \\
\hline 9 & $1.2385 \mathrm{e}-001$ & $2.0889 \mathrm{e}-002$ & $9.6870 \mathrm{e}-001$ & $1.0407 \mathrm{e}-002$ \\
\hline 10 & $6.2449 \mathrm{e}-002$ & $2.0641 \mathrm{e}-002$ & $9.7338 \mathrm{e}-001$ & $5.9795 \mathrm{e}-003$ \\
\hline 11 & $1.6847 \mathrm{e}-002$ & $2.2165 \mathrm{e}-002$ & $9.7512 \mathrm{e}-001$ & $2.7191 \mathrm{e}-003$ \\
\hline 12 & $1.7734 \mathrm{e}-003$ & $2.5830 \mathrm{e}-002$ & $9.7336 \mathrm{e}-001$ & $8.1170 \mathrm{e}-004$ \\
\hline 13 & $4.5269 \mathrm{e}-005$ & $3.1798 \mathrm{e}-002$ & $9.6809 \mathrm{e}-001$ & $1.1541 \mathrm{e}-004$ \\
\hline 14 & $1.3088 \mathrm{e}-007$ & $3.9920 \mathrm{e}-002$ & $9.6008 \mathrm{e}-001$ & - \\
\hline
\end{tabular}
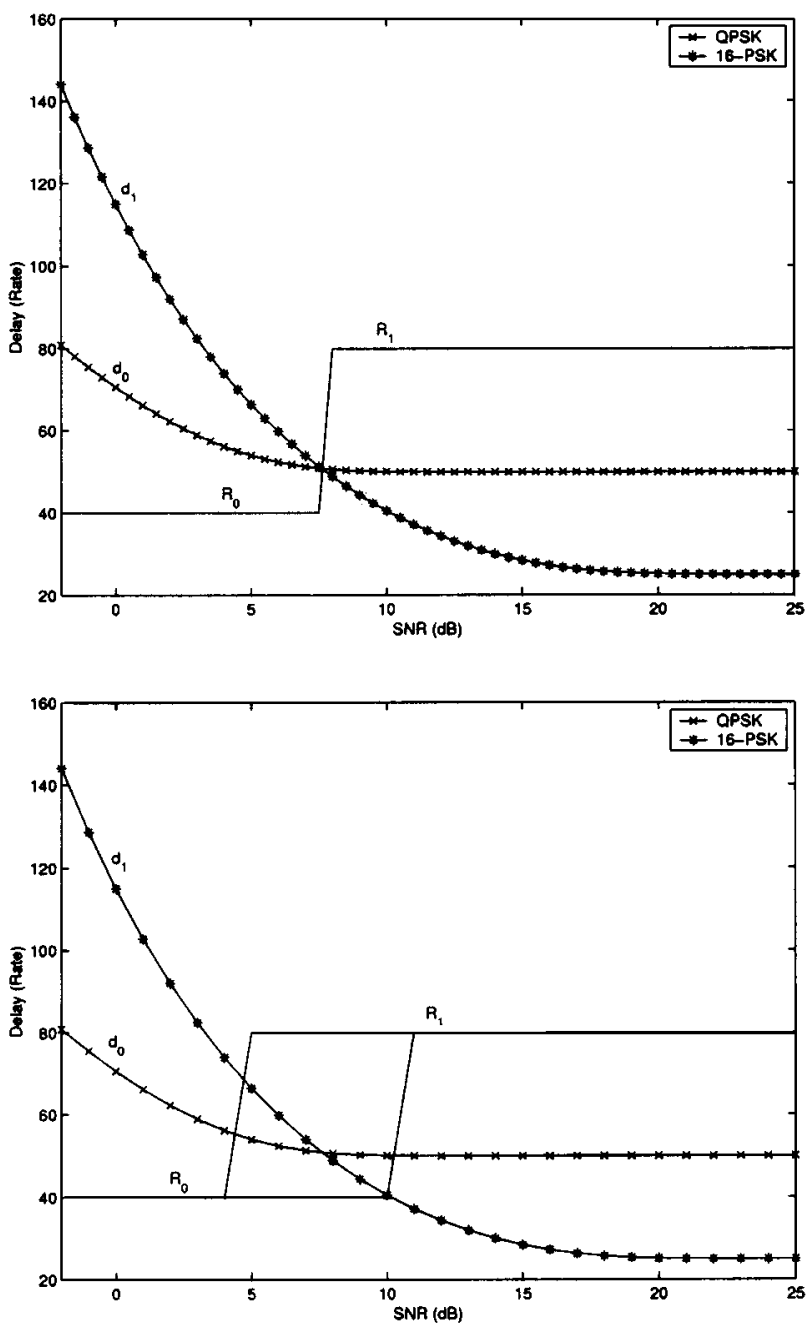

Fig. 6. Optimal rate control policy in a system with two admissible rates $R_{0}$, and $R_{1}$, in a Rayleigh fading channel, for $C_{s}=0$ (top) and $C_{s}=45$ (bottom).

becomes more sluggish. Our simulation results shown in Fig. 6 clearly supports this claim.

We should mention that both optimal policy and convergence rate of the value-iteration method depend on $\beta$. As $\beta$ decreases, 


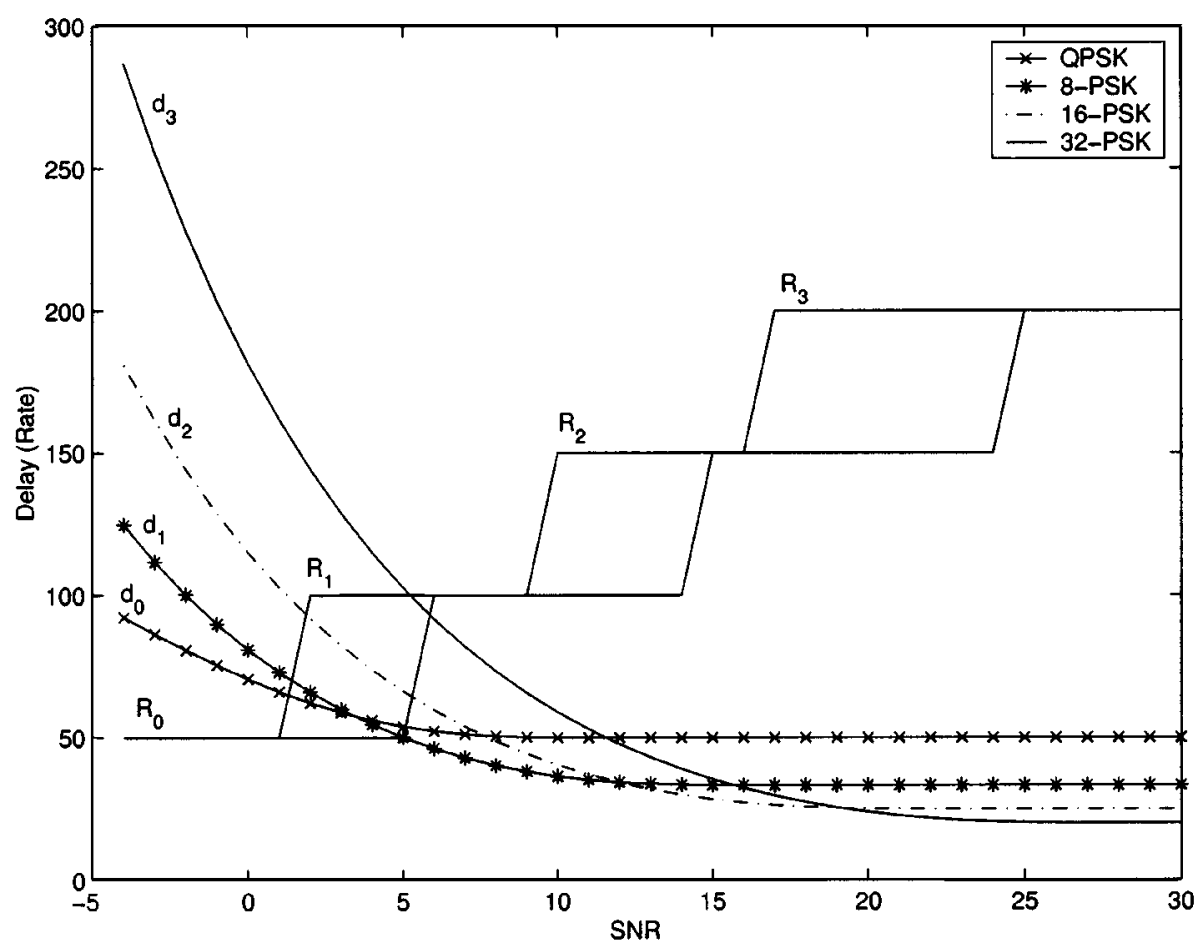

Fig. 7. Optimal rate control policy in a multirate system with four admissible rates $R_{0}, R_{1}, R_{2}$, and $R_{3}$ in a Rayleigh fading channel, for $C_{s}=40$.

TABLE II

Optimal Policy $\pi^{\star}(s, i)$ IN a System With Two Admissible Rate 0: $R_{0}$ (QPSK) AND RATE 1: $R_{1}$ (16-ARY PSK), FOR $C_{s}=45$

\begin{tabular}{c||c|c}
\hline state index $k$ & $\pi^{\star}\left(s_{k}, 0\right)$ & $\pi^{\star}\left(s_{k}, 1\right)$ \\
\hline \hline 0 & 0 & 0 \\
\hline 1 & 0 & 0 \\
\hline 2 & 0 & 0 \\
\hline 3 & 0 & 0 \\
\hline 4 & 0 & 1 \\
\hline 5 & 0 & 1 \\
\hline 6 & 0 & $\mathbf{1}$ \\
\hline 7 & 1 & $\mathbf{1}$ \\
\hline 8 & 1 & $\mathbf{1}$ \\
\hline 9 & 1 & 1 \\
\hline 10 & 1 & 1 \\
\hline 11 & 1 & 1 \\
\hline 12 & 1 & 1 \\
\hline 13 & 1 & 1 \\
\hline 14 & 1 & 1 \\
\hline
\end{tabular}

the value-iteration method converges faster to the final optimal rate control policy. A detailed analysis of the value-iteration method and the effect of $\beta$ on its convergence is provided in [17, Vol. II]. The effect of $\beta$ on the optimal policy is as follows: as $\beta$ decreases, the probability that the session is terminated in a time slot increases. Since in this case it would be more probable to terminate a session in the next time slot, the optimal policy tends to stay with the current rate for wider range of states (to avoid unnecessary switching costs, since it is more probable to end the session in next slot), i.e., the optimal policy becomes more sluggish. Consequently decreasing $\beta$ has a similar effect on optimal policy as increasing $C_{s}$. Since in practical systems $\beta$ is dictated by the traffic behavior in the system, $C_{s}$ which is a parameter in system design has to be adjusted accordingly. As a result, in our simulations we have fixed $\beta=0.8$, and only the effect of increasing $C_{s}$ is studied through simulations.

Next we consider the optimal rate control policy in multirate systems. So far we have only considered the optimal rate control policy for the systems with two admissible rates $R_{0}$ and $R_{1}$. The important feature of our proposed optimal rate control method is that, with a well-defined cost function, it can be generalized to more than two rates. Now we consider the cost function proposed in (12) and attempt to find the solution of (15). The next set of simulations is performed to obtain the optimal rate control policy for a system with $M=4$, i.e., there are four admissible rates $R_{0}, R_{1}, R_{2}$, and $R_{3}$ in the system. In this case, we assume that packets are transmitted in the downlink using one of the following four modulation schemes: QPSK, 8-PSK, 16-PSK, and 32-PSK modulations. The optimal rate control policy for such a system is illustrated in Fig. 7. As we expect again, the optimal rate control policy in a multirate system is also a threshold policy as shown in Fig. 7, where the delay curves $d_{0}, d_{1}, d_{2}$, and $d_{3}$, associated with each modulation scheme, are plotted in the same graph along with the optimal rate control policy (this way we can compare the thresholds against intersection points of delay graphs).

Finally, Figs. 8 and 9 illustrate how $\bar{D}_{\pi}$ and $\bar{S}_{\pi}$ vary as the switching cost $C_{s}$ increases. We assess the effectiveness of the proposed method by comparing the average delay $\bar{D}_{\pi}$ and expected number of rate switchings $\bar{S}_{\pi}$ for different values of the switching cost $C_{s}$. From Fig. $8, \bar{D}_{\pi}=210.5$ when $C_{s}=0$, and it is $\bar{D}_{\pi}=213.25$ when $C_{s}=45$, which represents only a $1.3 \%$ increase in average delay. On the other hand, from Fig. 9, $\bar{S}_{\pi}=0.235$ for $C_{s}=0$, and it is $\bar{S}_{\pi}=0.145$ for $C_{s}=45$, which represents more than a $38 \%$ decrease in rate switchings. 


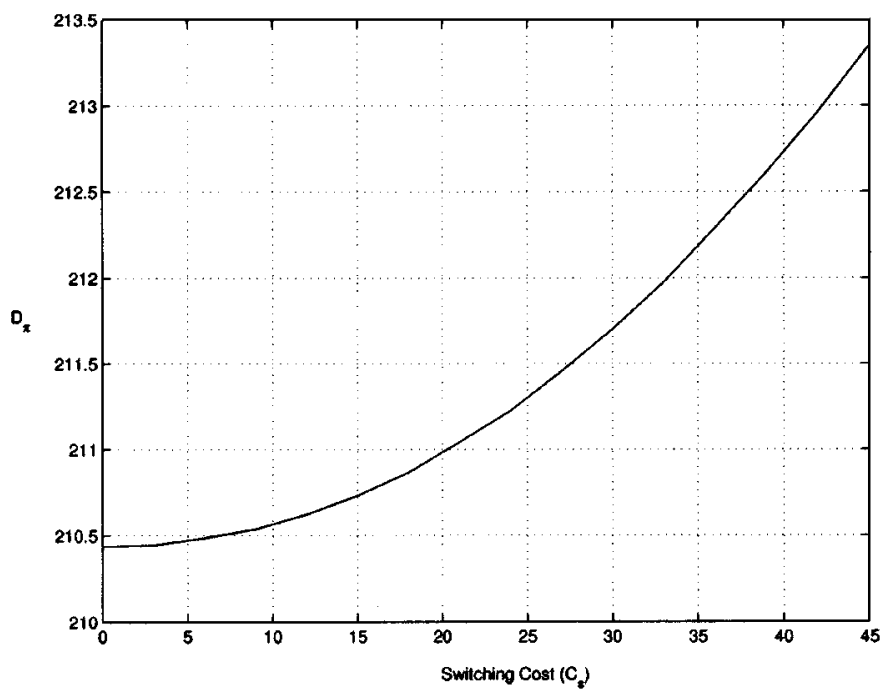

Fig. 8. Average delay $D_{\pi}$ versus rate switching $\operatorname{cost} C_{s}$.

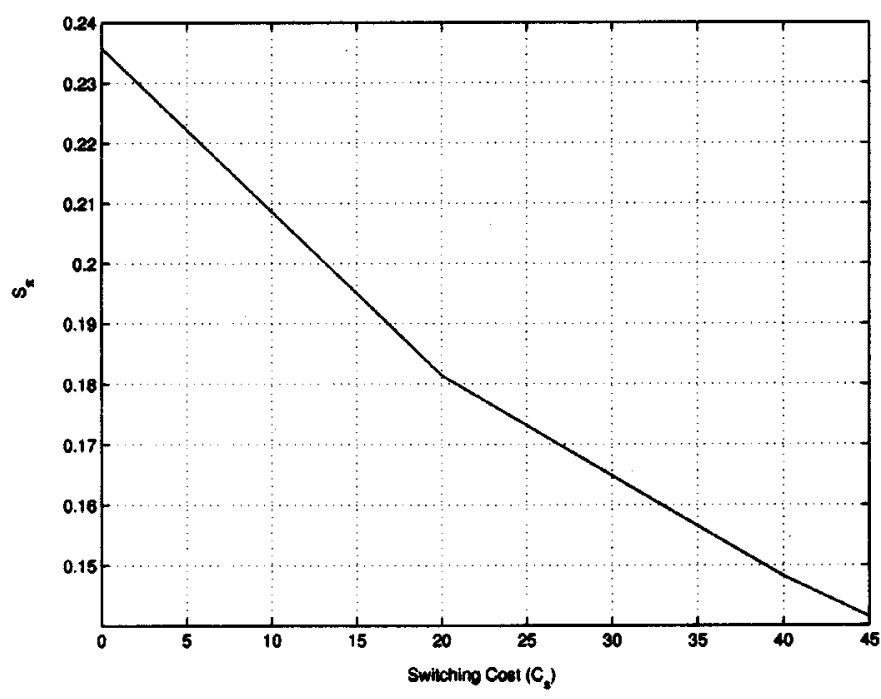

Fig. 9. Expected number of rate switchings $S_{\pi}$ versus rate switching $\operatorname{cost} C_{s}$.

Therefore, with sacrificing only a $1 \%$ increase in average delay in the system, we can save almost $40 \%$ in rate switchings.

\section{CONCLUSION}

In this paper we have studied the problem of optimal rate control in wireless networks with Rayleigh fading channels. A stochastic optimization technique based on the dynamic programming method was used to obtain the optimal rate control policy in such networks. Using the results from the theory of dynamic programming, it was shown that the optimal rate control policy is in the form of a threshold policy - a property of significance both from the analytical and implementation points of view. Simulation results confirmed that the optimal rate control policy is indeed a threshold policy. These results also demonstrated the effectiveness of our optimal rate control policy in optimizing the overall delay and number of rate switchings in the network. Simulation results indicate that by sacrificing only $1 \%$ of transmission quality in terms of the average delay one can achieve almost $40 \%$ reduction in rate switchings in the network.

\section{ACKNOWLEDGMENT}

The authors would like to thank the anonymous reviewers for valuable comments which significantly improved the presentation of this paper.

\section{REFERENCES}

[1] A. Ephremides and S. Verdu, "Control and optimization methods in communication network problems," IEEE. Trans. Automatic Control, vol. 34, pp. 930-942, September 1989.

[2] J. Razavilar, "Signal processing and performance analysis for optimal resource allocation in wireless networks," Ph.D. dissertation, University of Maryland, College Park, 1998.

[3] J. Razavilar, K. J. R. Liu, and S. I. Marcus, "Optimal rate control in wireless networks with fading channels," in Proc. VTC 99, IEEE Vehicular Tech. Conf., vol. 1, May 1999, pp. 807-811.

[4] N. Yin and M. G. Hluchyj, "A dynamic rate control mechanism for source coded traffic in a fast packet network," IEEE J. Select. Areas Commun., vol. 9, pp. 1003-1012, Sept. 1991.

[5] I. Lambadaris, P. Narayan, and I. Viniotis, "Optimal service allocation among two heterogeneous traffic types with no queueing," in Proc. 26th IEEE Conf. Decision and Control, Los Angeles, CA, Dec. 1987, pp. $1496-1498$.

[6] I. Lambadaris and P. Narayan, "Optimal control of arrivals at a blocking node," in Proc. Computer Networking Symp., Apr. 1988, pp. 209-213.

[7] D. T. Lee and F. P. Preparata, "Euclidean shortest path in the presence of rectilinear barriers," Networks, vol. 14, pp. 393-410, Oct. 1984.

[8] Y. Chang and E. Geraniotis, "Optimal policies for handoff and channel assignment in networks of LEO satellites using CDMA," Institute for Systems Research, University of Maryland, College Park, Tech. Rep. TR 96-27, 1996.

[9] Z. Rosberg, P. Varaiya, and J. Walrand, "Optimal control of service in tandem queues," IEEE Trans. Automat. Contr., vol. AC-27, pp. 600-610, June 1982.

[10] R. Rezaiifar, A. M. Makowski, and S. Kumar, "Stochastic control of handoffs in cellular networks," IEEE J. Select. Areas Commun., vol. 13, pp. 1348-1362, Sept. 1995.

[11] E. N. Gilbert, "Capacity of a burst-noise channel," Bell Syst. Tech. J., vol. 39, pp. 1253-1265, Sept. 1960.

[12] E. O. Elliot, "Estimates of error rates for codes on burst-noise channels," Bell Syst. Tech. J., vol. 42, pp. 1977-1997, Sept. 1963.

[13] H. S. Wang and N. Moayeri, "Modeling, capacity, and joint source/channel coding for Rayleigh fading channels," in Proc. IEEE Vehicular Technology Conf., May 1993, pp. 473-479.

[14] _ _ "Finite-state Markov channel_A useful model for radio communication channels," IEEE Trans. Veh. Technol., vol. 44, pp. 163-170, Feb. 1995.

[15] Q. Zhang and S. A. Kassam, "Finite-state Markov model for Rayleigh fading channels," IEEE Trans. Commun., vol. 47, pp. 1688-1692, Nov. 1999.

[16] M. L. Puterman, Markov Decision Processes, Discrete Stochastic Dynamic Programming. New York: Wiley, 1994.

[17] D. P. Bertsekas, Dynamic Programming and Optimal Control: Athena Scientific, 1995.

[18] S. M. Ross, Stochastic Dynamic Programming. New York: Academic, 1983.

[19] P. P. Varaiya, Notes on Optimization. New York: Van Nostrand-Reinhold, 1972.

[20] R. G. D. Bertsekas, Data Networks. Englewood Cliffs, NJ: PrenticeHall, 1992.

[21] J. G. Proakis, Digital Communications, 3rd ed. New York: McGrawHill, 1995. 


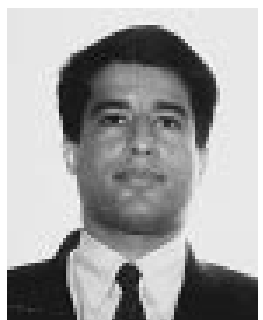

Javad Razavilar (S'89-M'99) received the B.S. degree in electrical engineering from Sharif University of Technology, Tehran, Iran, in 1989 and the M.S. and $\mathrm{Ph} . \mathrm{D}$. degrees in electrical engineering from the University of Maryland at College Park in 1996 and 1998 , respectively.

From January 1999 to January 2001, he was with the Technology Development Center of 3Com Corporation in San Diego, CA, where he was a Senior Member of Technical Staff, working on the design and development of communications algorithms. At 3Com Corporation, he was also leading the activities with Center for Wireless Communications (CWC) at University of California San Diego (UCSD), where he was a Visiting Research Scientist in Electrical and Computer Engineering Department of UCSD. Since January 2001, he has been with Magis Networks, Inc., a start-up company in San Diego, CA, as a System Engineer, where he is designing innovative algorithms for advanced wireless multimedia communication chipsets based on $802.11 \mathrm{a} / \mathrm{HIPERLAN2}$ standards. His research interests include stochastic optimization, with applications to wireless communications, statistical signal processing, digital communications, and communication networks.

Dr. Razavilar is a member of Eta Kappa Nu, Gamma Xi chapter. He is also a Member of the IEEE Information Theory, Communications, and Signal Processing Societies.

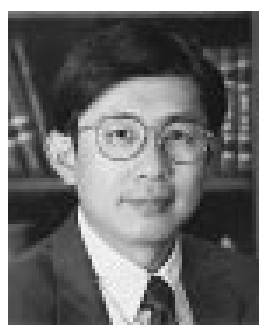

K. J. Ray Liu (S'86-M'90-SM'93) received the B.S. degree from the National Taiwan University, Taiwan, R.O.C., and the Ph.D. degree from the University of California at Los Angeles, both in electrical engineering.

$\mathrm{He}$ is a Professor of Electrical and Computer Engineering Department and Institute for Systems Research of University of Maryland, College Park. His research interests span broad aspects of signal processing algorithms and architectures, image/video compression, coding, and processing, wireless communications, security, and medical and biomedical technology in which he has published over 200 papers.

Dr. Liu received numerous awards including the 1994 National Science Foundation Young Investigator, the IEEE Signal Processing Society's 1993 Senior Award (Best Paper Award), IEEE Vehicular Technology Conference Best Paper Award, Amsterdam, 1999, and the George Corcoran Award in 1994 for outstanding contributions to electrical engineering education and the Outstanding Systems Engineering Faculty Award in 1996 in recognition of outstanding contributions in interdisciplinary research, both from the University of Maryland. He is Editor-in-Chief of EURASIP Journal on Applied Signal Processing, and has been an Associate Editor for the IEEE Transactions on Signal Processing, a Guest Editor of special issues on Multimedia Signal Processing of ProceEdINGS OF THE IEEE, a Guest Editor for a special issue on Signal Processing for Wireless Communications of the IEEE JouRnAl OF SELECTED AREAS IN COMMUNICATIONS, a Guest Editor for a special issue on Multimedia Communications over Networks of the IEEE Signal Processing Magazine, a Guest Editor of a special issue on Multimedia over IP of the IEEE TRANSACTIONS ON MULTIMEDIA, and an editor of the Journal of VLSI Signal Processing Systems. He currently serves as the Chair of Multimedia Signal Processing Technical Committee of the IEEE Signal Processing Society and the series editor of Marcel Dekker series on signal processing and communications.

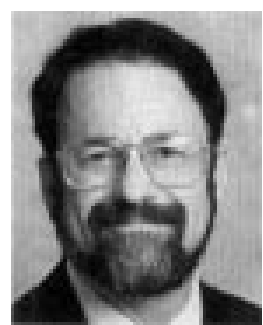

Steven I. Marcus (S'70-M'75-SM'80-F'86) received the B.A. degree in electrical engineering and mathematics from Rice University, Houston, TX, in 1971 and the S.M. and Ph.D. degrees in electrical engineering from the Massachusetts Institute of Technology, Cambridge, in 1972 and 1975, respectively.

From 1975 to 1991, he was with the Department of Electrical and Computer Engineering at the University of Texas at Austin, where he was the L.B. (Preach) Meaders Professor in Engineering. He was Associate Chairman of the Department during the period 1984-1989. In 1991, he joined the University of Maryland, College Park, where he was Director of the Institute for Systems Research until 1996. He is currently a Professor in the Electrical and Computer Engineering Department and the Institute for Systems Research at the University of Maryland, and Chair of the Electrical and Computer Engineering Department. Currently, his research is focused on stochastic control and estimation, with applications in semiconductor manufacturing, telecommunication networks, and preventive maintenance. He is Editor-in-Chief of the SIAM Journal on Control and Optimization 